\title{
Real-Time Data-Reusing Adaptive Learning of a Radial Basis Function Network for Tracking Evoked Potentials
}

\author{
Wei Qiu*, Member, IEEE, Chunqi Chang, Wenqing Liu, Paul W. F. Poon, Yong Hu, F. K. Lam, Roger P. Hamernik, \\ Gang Wei, and Francis H. Y. Chan
}

\begin{abstract}
Tracking variations in both the latency and amplitude of evoked potential (EP) is important in quantifying properties of the nervous system. Adaptive filtering is a powerful tool for tracking such variations. In this paper, a data-reusing nonlinear adaptive filtering method, based on a radial basis function network (RBFN), is implemented to estimate EP. The RBFN consists of an input layer of source nodes, a single hidden layer of nonlinear processing units and an output layer of linear weights. It has built-in nonlinear activation functions that allow learning of function mappings. Moreover, it produces satisfactory estimates of signals against a background noise without $a$ priori knowledge of the signal, provided that the signal and noise are independent. In clinical situations where EP responses change rapidly, the convergence rate of the algorithm becomes a critical factor. A carefully designed data-reusing RBFN can accelerate the convergence rate markedly and, thus, enhance its performance. Both theoretical analysis and simulation results support the improved performance of our new algorithm.
\end{abstract}

Index Terms-Convergence rate, data-reusing algorithm, evoked potential, radial basis function network, tracking ability.

\section{INTRODUCTION}

$\mathbf{E}$ VOKED potentials (EPs) represent gross electrical activity of the brain usually generated by sensory stimulation. The amplitude of the EP signal is often lower than the background noise [i.e., mostly ongoing electroencephalography (EEG)]. Ensemble averaging (EA) is the most widely used method for detecting components of the EP. EA sums the responses to repetitive stimulus presentations in order to obtain

Manuscript received August 27, 2004; revised May 15, 2005. This work was supported in part by the National Institute for Occupational Safety and Health under Grant 1-R03-OH008175-01 and Grant 1-R01-OH007801-01A1, in part by the National Natural Science Foundation of China, under Grant 69871011, and in part by the Research Grant Council under Grant HKU 553/96M, Grant HKU 7036/97E, Grant CUHK 4260/99M, Grant HKU CRCG 04/05, and Grant N_HKU703/03, and in part by the National Science Council, Taiwan. Asterisk indicates corresponding author.

*W. Qiu is with the Auditory Research Laboratory, State University of New York, Plattsburgh, USA. He is also with the School of Electronic and Information Engineering, South China University of Technology, 310007 Guangzhou, China (e-mail: wei.qiu@plattsburgh.edu).

C. Chang, W. Liu, F. K. Lam, and F. H. Y. Chan are with the Department of Electrical and Electronic Engineering, University of Hong Kong, Hong Kong.

P. W. F. Poon is with the Department of Physiology, College of Medicine, National Cheng Kung University, Tainan 701, Taiwan, R.O.C.

$\mathrm{Y}$. Hu is with the Department of Orthopaedic Surgery, University of Hong Kong, Hong Kong.

R. P. Hamernik is with the Auditory Research Laboratory, State University of New York, Plattsburgh, NY 12901 USA.

G. Wei is with the School of Electronic and Information Engineering, South China University of Technology, 310007 Guangzhou, China

Digital Object Identifier 10.1109/TBME.2005.862540
EP estimates with an acceptable signal-to-noise ratio (SNR). However, studies showed that EP signals could often be nonstationary and, therefore, have characteristics that could vary from trial to trial [1]-[5]. Such across-trial response variability will be failed to be revealed using EA.

Much effort has been made to track trial-to-trial variations in the EP to improve SNR and to speed up measurement. Woody [6] described a procedure for computing averages from single EP in which the latency variation of various components in the EP was aligned by using a cross-correlation function. Aunon and McGillem [7] introduced a method called the latency corrected average using a minimum mean square error (MMSE) filter to extract individual components in the EPs. Cerutti and associates [8] also proposed a parametric method of identifying a single-trial EP. All these methods improved the EP results to some extent.

Adaptive filtering has the ability to adjust its own parameter settings automatically, and requires little or no a priori knowledge of the characteristics of the signal or noise. Since the EP is a time-varying signal, adaptive filtering appears to be appropriate for its estimation. Recently the technique of adaptive filtering has been applied with success to enhance the EP estimates [9]-[16].

Considering the possible nonlinear nature of the system, neural networks for both linear and nonlinear systems are potentially useful in determining response variability. Recently, in many areas of signal processing there has been a growing use of neural networks with built-in nonlinear processing elements [17], [18]. In this paper, an algorithm of data-reusing radial basis function network (DR-RBFN) was used to improve the convergence performance of an approach of RBFN reported earlier [19]. During each iteration the data-reusing algorithm reuses the current (or previous) desired response and data vector to update the adaptive tap-weight vector multiple times [20], [21]. Such data-reusing LMS algorithms have been reported to show a dramatic improvement in the convergence rate over the traditional method of LMS [20], [22]. When carefully designed, DR-RBFN can markedly accelerate the convergence rate and, therefore, results in better performance. Both theoretical analysis and simulation results supported improved performance over the previous RBFN algorithm reported earlier [19].

\section{METHODS}

\section{A. Basics of RBFN for EP Estimation}

As shown in Fig. 1, the RBFN is a multilayer feedforward neural network consisting of an input layer of source nodes, a layer of nonlinear hidden units that operate as kernel nodes and 


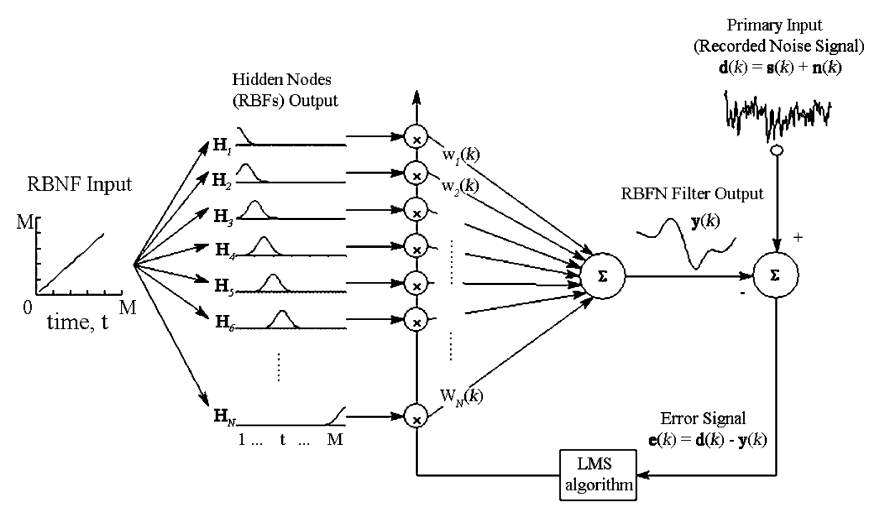

Fig. 1. Schematic of the adaptive Gaussian RBFN used for EP estimation.

an output layer of linear weights. In response to an input vector, $\mathbf{x}$, the hidden node outputs, $h_{j}(\mathbf{x})$, computed by the hidden layer are linearly combined to form the network response that is processed in combination with a desired response presented to the output layer. The weights are trained in a supervised fashion using an appropriate linear learning method.

Assume we have $K$ trials of EP signal, and each trial contains $M$ points of data, denoted as

$$
\mathbf{d}(k)=\left[\begin{array}{lll}
d_{1}(k) & \cdots & d_{M}(k)
\end{array}\right]^{T}, \quad k=1, \ldots, K .
$$

With $\mathbf{x}$ as input, the output of a RBFN that has $N$ hidden nodes will be

$$
\mathbf{y}(k)=\sum_{j=1}^{N} w_{j}(k) h_{j}(\mathbf{x})=\mathbf{H w}(k)
$$

where

$$
\mathbf{w}(k)=\left[\begin{array}{lll}
w_{1}(k) & \cdots & w_{N}(k)
\end{array}\right]^{T}
$$

and

$$
\mathbf{H}=\left[\begin{array}{cccc}
h_{1}\left(x_{1}\right) & h_{2}\left(x_{1}\right) & \cdots & h_{N}\left(x_{1}\right) \\
h_{1}\left(x_{2}\right) & h_{2}\left(x_{2}\right) & \cdots & h_{N}\left(x_{2}\right) \\
\vdots & \vdots & \ddots & \vdots \\
h_{1}\left(x_{M}\right) & h_{2}\left(x_{M}\right) & \cdots & h_{N}\left(x_{M}\right)
\end{array}\right]
$$

The overall objective is to minimize the error signal

$$
\mathbf{e}(k)=\mathbf{d}(k)-\mathbf{y}(k)=\mathbf{d}(k)-\mathbf{H w}(k) .
$$

Using the famous LMS algorithm the weight vector $\mathbf{w}$ is adapted once every trial by the steepest-descent optimization

$$
\mathbf{w}(k+1)=\mathbf{w}(k)+\mu \mathbf{H}^{T} \mathbf{e}(k)
$$

where $\mu$ is the step size which regulates the speed and stability of adaptation.

In a previous paper [19], a Gaussian function was used as the activation function for the hidden nodes since the function is bounded and stable in adaptive processing. The RBF produces a localized response to the input stimulus. The response is related to the Euclidean distance between the input and the centroid associated with the basis function. Each node produces an identical output for inputs that lie at a fixed radial distance from its center, $\mathbf{c}_{j}$

$$
\text { Gaussian RBF }: h_{j}(\mathbf{x})=\exp \left(-\frac{\left\|\mathbf{x}-\mathbf{c}_{j}\right\|^{2}}{\boldsymbol{\sigma}_{j}^{2}}\right)
$$

where $\boldsymbol{\sigma}_{j}$ is the variance. Considering EP signals having a timelocked feature, a simple but effective method was used to design a real-time EP tracking RBFN [19]. Assume each recorded trial of the EP signal has $M$ data points, the input of the RBF could be simply designed as $x=t(1 \leq t \leq M)$. Therefore, the Gaussian radial basis function $h_{j}(k)$ could be written as

$$
h_{j}(t)=\exp \left(-\frac{\left(t-c_{j}\right)^{2}}{\sigma_{j}^{2}}\right) \quad j=1,2, \ldots, N
$$

where $c_{j}$ and $\sigma_{j}$ are the center and the width of the $j$ th RBF respectively. It has been shown [19] that the following simple design of $c_{j}$ and $\sigma_{j}$ is effective for tracking EP signals:

$$
c_{j}=(j-1)\left(\frac{M-1}{N-1}\right)+1, \quad \text { for } j=1 \text { to } N
$$

where $M$ is the number of data points in each trial and $N$ indicates the number of hidden neurons. The RBF was design to have the same width, i.e.,

$$
\sigma=\beta \frac{M-1}{N-1}
$$

where $\beta$ is set at 0.8 . Notice that, if the center and width of the RBFs are determined by (9) and (10), matrix $\mathbf{H}$ becomes a constant. A constant $\mathbf{H}$ is of special interest since it makes processing in real-time possible using our new algorithm.

The primary input at the $i$ th iteration is a trial of the recorded signal denoted by the vector $\mathbf{d}$. Once the hidden layer is designed, the next step is to develop an algorithm to adjust the weight vector $\mathbf{w}(k)=\left[\begin{array}{lll}w_{1}(k) & \cdots & w_{N}(k)\end{array}\right]^{T}$ ( $k$ is the trial number) such that the network output $\mathbf{y}(k)$ estimates the underlying EP signal $\mathbf{s}(k)$. In (7), the hidden layer output matrix, $\mathbf{H}$, acts as the input for the conventional linear adaptive filter. Thus, an adaptive LMS algorithm can be used to adjust the weight vector.

\section{B. Normalized Data-Reusing LMS (NDR-LMS) Algorithm}

Widrow's LMS algorithm [23] is one of the most widely used approaches for practical applications. This algorithm is an approximation of the steepest descent optimization strategy using the instantaneous squared error at each iteration to estimate the mean square error. It is simple and easy to implement in real-time. However, the convergence rate of the LMS algorithm might be rather slow when the input signal is colored. Some methods are available to improve the convergence rate. Techniques such as pseudorandom modulation [24], or GaussNewton optimization [25] could be used to decorrelate the input signal. A recursive least squares (RLS) algorithm is another choice, which has a faster convergence rate but with additional complexity in computation. In this paper, a simpler and effective method, called the normalized data-reusing LMS (NDR-LMS) algorithm is applied.

The normalized LMS algorithm could be derived by the principle of minimal disturbance [26]. For our specific problem (for its derivation please see Appendix ), the normalized LMS algorithm is as follows:

$$
\mathbf{w}(k+1)=\mathbf{w}(k)+\mu \mathbf{H}^{T}\left(\mathbf{H H}^{T}+\varepsilon \mathbf{I}\right)^{-1} \mathbf{e}(k)
$$

where $\mu$ is the step size which regulates the speed and stability of adaptation, and $\varepsilon$ is a small positive constant used to realize the pseudoinverse of the matrix. 
The NDR-LMS algorithm is a modification of the normalized LMS algorithm described above, which reuses data pairs $(\mathbf{H}, \mathbf{d})$ from previous iterations to generate the new gradient estimates that are in turn used to update the adaptive weight vector [20]. Thus, the NDR-LMS algorithm is as following:

$$
\mathbf{w}_{k}(l+1)=\mathbf{w}_{k}(l)+\mu \mathbf{H}^{T}\left(\mathbf{H} \mathbf{H}^{T}+\varepsilon \mathbf{I}\right)^{-1} \mathbf{e}_{k}(l)
$$

where

$$
\mathbf{e}_{k}(l)=\mathbf{d}(k-l)-\mathbf{H w}_{k}(l) \text { for } l=0, \ldots, L .
$$

In this algorithm, a number of $L$ previous trials, in addition to the current trial, are reused to estimate the current weight vector. Thus, we have

$$
\mathbf{w}_{k}(0)=\mathbf{w}(k), \quad \mathbf{w}(k+1)=\mathbf{w}_{k}(L+1) .
$$

For each trial $k$, the data are reused sequentially, and at each step of the data reusing process the intermediate weight vector, denoted by $\mathbf{w}_{k}(l)$ with the sub-index $k$ as the trial number, is estimated by the normalized LMS algorithm. Then, the intermediate weight vector obtained at the last data reusing step, $\mathbf{w}_{k}(L+1)$, is assigned as the estimate of $\mathbf{w}(k+1)$. Here $L$ denotes the number of trials reused, and $\mathbf{d}(k-l), l=0, \ldots, L$, are the reused trials for the estimation of $\mathbf{w}(k+1)$.

Comparing to (6) the algorithm has a factor $\left(\mathbf{H H}^{T}+\varepsilon \mathbf{I}\right)^{-1}$ which accounts for decorrelation for the reference input. Decorrelation will speed up the convergence. Therefore, the algorithm benefits from the decorrelation factor.

The convergence improvement in the NDR-LMS algorithm can be explained using the geometric interpretation of Alexander [27] and Schnaufer and Jenkins [28]. In the LMS algorithm, the instantaneous error surface is expressed as

$$
\|\mathbf{e}(k)\|^{2}=\|\mathbf{d}(k)\|^{2}-2 \mathbf{w}^{T}(k) \mathbf{H}^{T} \mathbf{d}(k)+\mathbf{w}^{T}(k) \mathbf{H}^{T} \mathbf{H w}(k) .
$$

In (14), the constant matrix $\mathbf{H}$, consisting of the Gaussian kernels, represents the deterministic reference input of the adaptive filter, $\mathbf{d}(k)$ represents the EP trials, which is the primary input of the adaptive filter. If the EP trials are stationary, the expected value of (14) is the true mean square error (MSE) surface. Therefore, minimizing this equation with the steepest descent algorithm is expected to converge to the Wiener solution.

Noting that only when $\mathbf{e}(k)=0,\|\mathbf{e}(k)\|^{2}$ reaches its minimum value (zero). With $\mathbf{e}(k)=0$ from (5) we have

$$
\mathbf{H w}(k)=\mathbf{d}(k) \text {. }
$$

The solution set $\mathbf{w}(k)$ is a hyperplane on which $\|\mathbf{e}(k)\|^{2}$ is minimized. Since the input vector $\mathbf{H}$ parametrizes the hyperplane, this vector is perpendicular to the solution set. Defining $\mathbf{s}(k)$ as the solution hyperplane at iteration $k$, (13) can be expressed as a vector orthogonally projected onto $\mathbf{s}(k)$ by subtracting from it the appropriate scalar multiple of $\mathbf{H}$. It is interesting to note that $\mathbf{w}(k+1)$ will lie in $\mathbf{s}(k)$, as shown in Fig. 2(b). If the normalized LMS algorithm is applied, it only takes one step directly toward the solution hyperplane, which explains why the normalized LMS algorithm converges faster than the LMS algorithm. Fig. 2(a) illustrates the geometric interpretation of the normalized LMS at a single iteration.

In the NDR-LMS algorithm, since a different pair of input $(\mathbf{d}(k), \mathbf{H})$ is used to update the weight vector at each step of

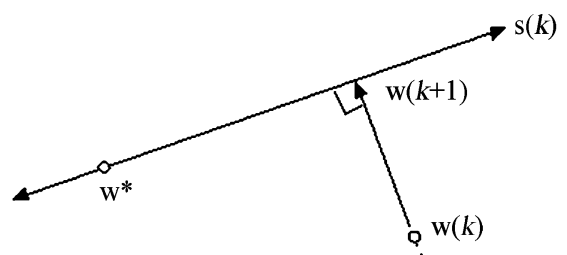

(a)

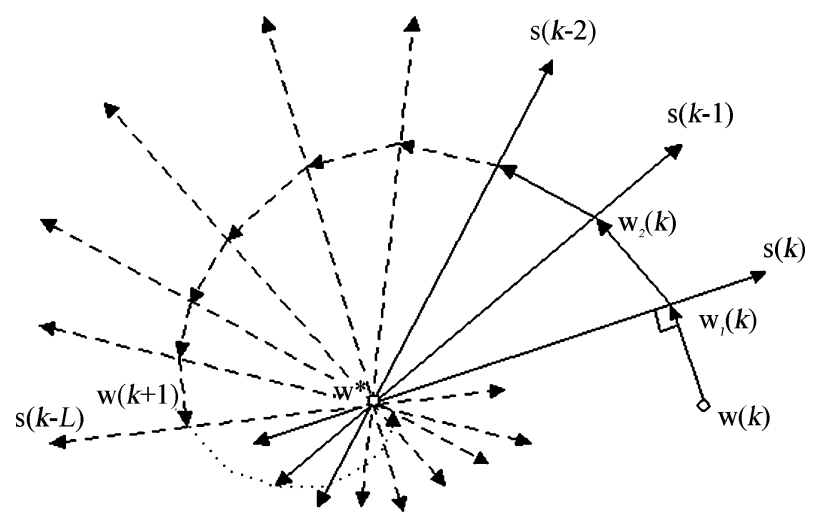

(b)

Fig. 2. Geometric interpretation illustrating the convergence improvement in (a) conventional normalized LMS, and (b) NDR-LMS.

the iteration in a different direction, a faster convergence is expected. Note that in the NDR-LMS algorithm in (12) and (13), it is the new data pair that is used at the beginning of a new iteration for the first weight update. New data points are used because they contain new information and should, therefore, provide the best update direction [20]. Fig. 2(b) demonstrates how the NDR-LMS converges to its Wiener solution $\mathrm{w}^{*}$ if the input signal is stationary. It is clear from Fig. 2(b) that each operation for $L-1$ pairs of data being reused gets $L-1$ steps closer to $\mathrm{w}^{*}$, thus, will speed up the convergence rate. Moreover, since only previous pairs of data are reused, the algorithm can operate in a real-time situation. For sufficiently large values of $L$ the system will converge rapidly. However, when the signal is nonstationary (i.e., time-varying), the optimal $\mathrm{w}^{*}$ is no longer constant over time. NDR-LMS can also be used for nonstationary signal tracking. With careful selection of $L$ this method is particularly useful in the case of on-line EP signal estimation. Note that the term $\mathbf{H}^{T}\left(\mathbf{H H}^{T}+\varepsilon \mathbf{I}\right)^{-1}$ in (12) is constant when the center and width of the RBFs are determined by (9) and (10). Thus, the DR-RBFN is easily approached in real-time with only $L-1$ additional operations required for each iteration.

The NDR-LMS algorithm in the paper is updated blockwise, i.e., the adaptive weight vector is updated in the form of trial. The solution hyperplanes at iteration $k$ and $k-1$ can be obtained by using (5) given $\mathbf{e}(k)=0$, i.e.,

$$
\begin{aligned}
\mathbf{s}(k) & : \mathbf{H w}(k)=\mathbf{d}(k) \\
\mathbf{s}(k-1) & : \mathbf{H w}(k)=\mathbf{d}(k-1) .
\end{aligned}
$$

Although the reference input matrix $\mathbf{H}$ is deterministic, solution hyperplanes $\mathbf{s}(k)$ is not parallel to $\mathbf{s}(k-1)$. Since vector $\mathbf{d}(k)$ is not parallel to vector $\mathbf{d}(k-1)$, where $\mathbf{d}(k)$ is the $k$ th EP recording trial. Thus, the block NDR-LMS can be effectively used for EP signal estimation. 


\section{Real-Time Implementation of the NDR-LMS Algorithm for EP Estimation}

For the NDR-LMS algorithm to estimate the EP in real-time, computational efficiency is very important. The batch mode approach was used to update the weights of the RBFN. In the batch mode approach, the weight adjustment is only performed after all data points of each trial have been presented. EP data recording is usually triggered by a certain stimulus. To estimate the EP in real-time, the NDR-LMS algorithm with the last $L$ trials can be implemented during the interval between the current and the subsequent stimulus. For EP estimation, the selection of the value for $L$ is particularly important in the DNR-LMS algorithm. The selection of $L$ is a twofold problem. First, the larger the $L$, the faster is the convergence rate (see Fig. 2). On the other hand, the value for $L$ is limited by interstimulus interval. Thus, in the selection of $L$ the following need to be considered, viz., the interstimulus interval, the nature of the EP signal and the computer hardware used. We found empirically that setting $L=3 \sim 5$ is adequate for real-time EP tracking in most situations we had tested. Note that the term $\mathbf{H}^{T}\left(\mathbf{H} \mathbf{H}^{T}+\varepsilon \mathbf{I}\right)^{-1}$ in (11) is a constant matrix when the center and width of the RBFs are determined by (9) and (10). Thus, only a fixed array is needed to save this constant matrix. Implementation of the NDR-LMS algorithm requires additional memory to save the last $L$ trials of the EP recordings. With powerful digital signal processing (DSP) chips or computer systems currently available, the DR-RBFN is easily approached in real-time with only $L-1$ additional operations required for each epoch.

\section{Simulations AND RESUlts}

A simulated brainstem auditory evoked potential (BAEP) signal is shown in Fig. 3(a). It was obtained by averaging 1000 trials of surface recorded EP data from a human subject, and mixed with an ongoing EEG signal simulated by the following autoregressive model [12]:

$$
\begin{gathered}
E E G_{i}(k)=1.508 E E G_{i}(k-1)-0.1587 E E G_{i}(k-2) \\
-0.3109 E E G_{i}(k-3)-0.0510 E E G_{i}(k-4)+n(k)
\end{gathered}
$$

where $n(k)$ is a driven white noise process with a Gaussian distribution, and the simulated EEGs were uncorrelated across trials. Fig. 3(b) shows one trial of the stimulated ongoing EEG.

The performance of the RBFN depends primarily on the number of RBFs used. A Large $N$ will increase the approximation capability of the RBFN but could also decrease the ability to track signal variations. A tradeoff must be made to select a proper number of RBFs. Fung and associates [19] found that it was suitable to set $N=30$ for BAEP signal estimation using the RBFN.

Theoretical analysis of the tracking performance of an adaptive algorithm is very complicated if not impossible. This kind of analysis is beyond the scope of this paper. However, some related simulations can be done to check the tracking performance of our new algorithm. Concerning of the influence of noise, two simulations were conducted in which both noise levels' amplitude and latency variations are taken into account.

\section{A. Tracking Performance Simulation 1 (Gradual Variations)}

To compare the performance of the previous RBFN [19] and the data-reusing algorithms for nonstationary signals, a total of
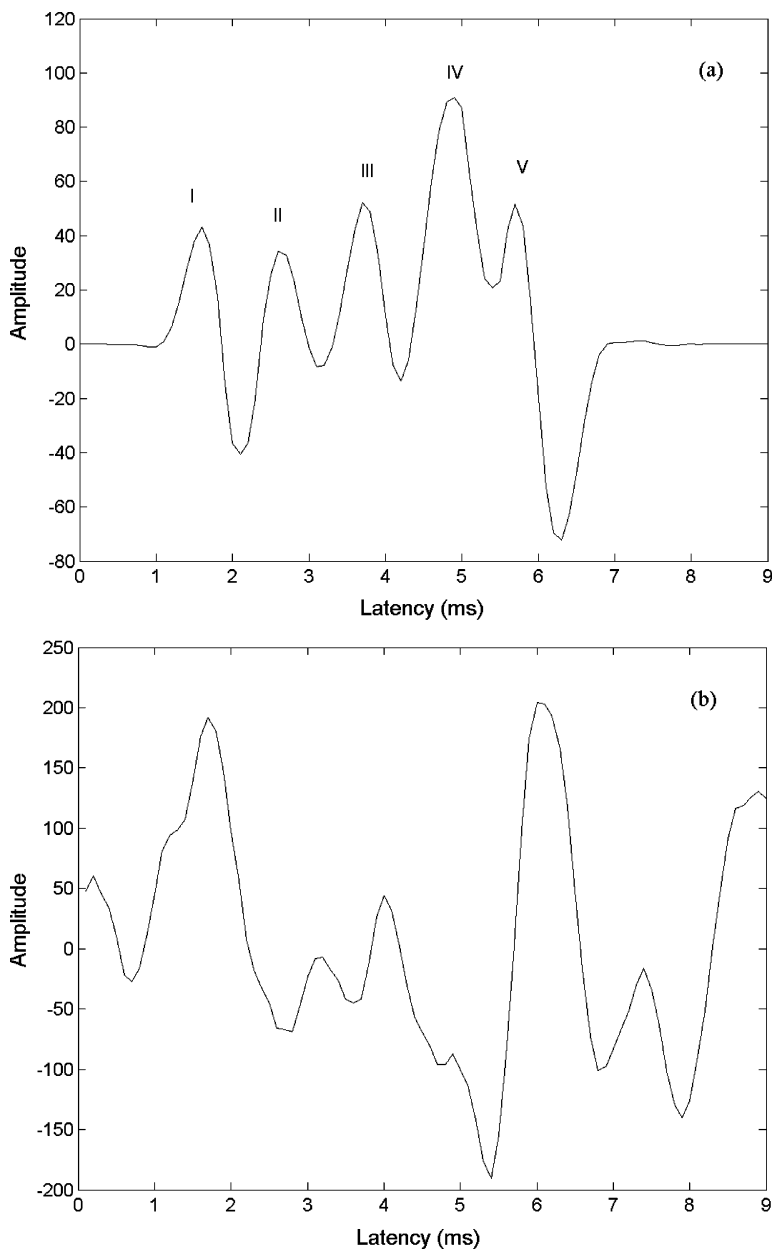

Fig. 3. Simulated data: (a) BAEP signal; (b) one trial of the ongoing EEG signal. The various peak latencies in the simulated BAEP are marked I to $\mathrm{V}$ according to convention.

400 trials of a simulated EP response were generated by adding the simulated EP signal with both latency and amplitude variations to the simulated EEG at a SNR of $-5 \mathrm{~dB}$. Each trial contains 90 data points. To simulate the variations in amplitude, the signal amplitudes of three selected sections from the 400 trials were amplified. The latency of the simulated EP signal shifted progressively in a sinusoidal manner over a range of 1 ms (compared with a EP response duration of $6 \mathrm{~ms}$ ). Fig. 4(a) shows the simulated EP signal with latency and amplitude variations, while Fig. 4(b) is the simulated raw EP recordings with $\mathrm{SNR}=-5 \mathrm{~dB}$.

Given that EP signals can be nonstationary, it is important to speed up the algorithm's convergence rate. The performance of a normalized data-reusing LMS algorithm for the RBFN was studied in this simulation. Three different numbers of previous trials ( $L=0,3$, and 5) were used to update the weight matrix according to (9) and (10). Fig. 5 shows the MSE curves of the NDR-LMS algorithm using three different values of $L$. It is evident from Fig. 5 that NDR-LMS markedly improves tracking performance, in comparison with conventional normalized LMS $(L=0)$. The NDR-LMS algorithm with a larger $L$ could yield more precise estimates of stationary signals [20]. However, for nonstationary signals this may not always be true since the signal changes over time, the solution hyperplane of the weights might also change. Thus, a large $L$ may introduce 

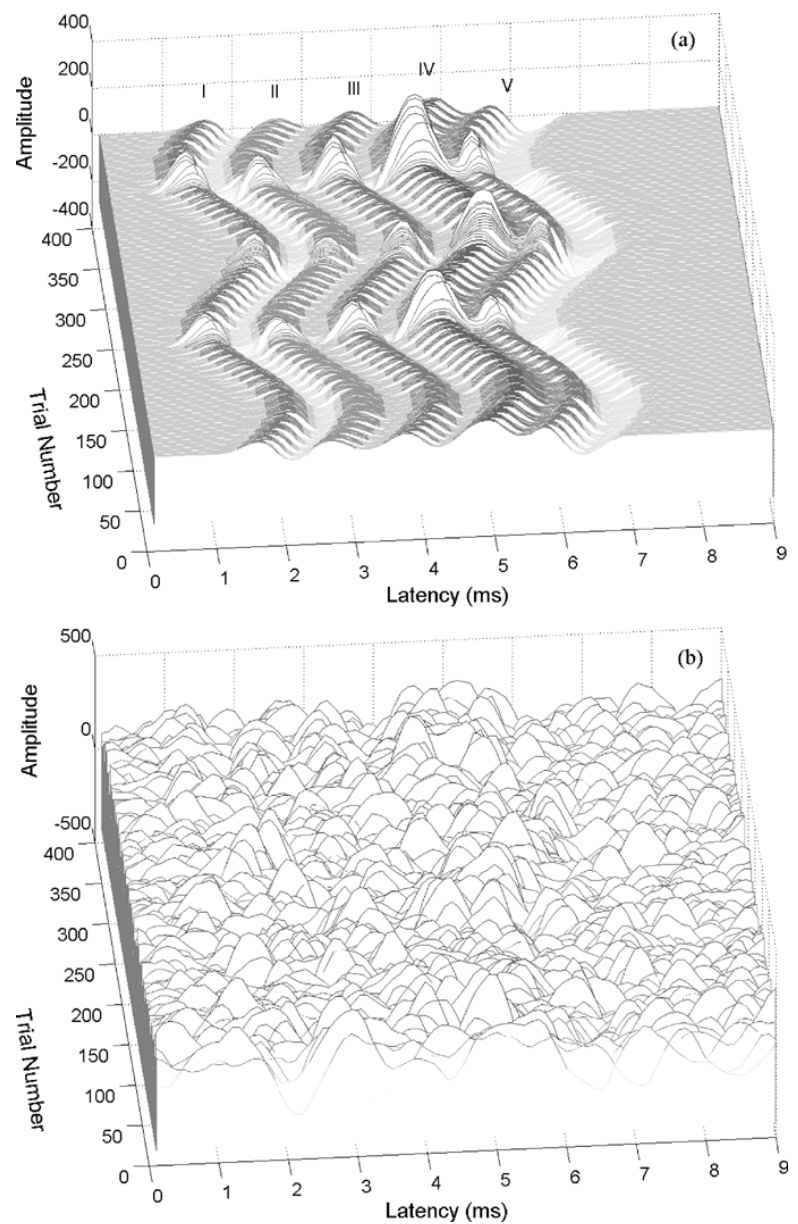

Fig. 4. Simulated BAEP signal with artificially introduced latency and amplitude variations (a), and raw EP data at $\mathrm{SNR}=-5 \mathrm{~dB}$.

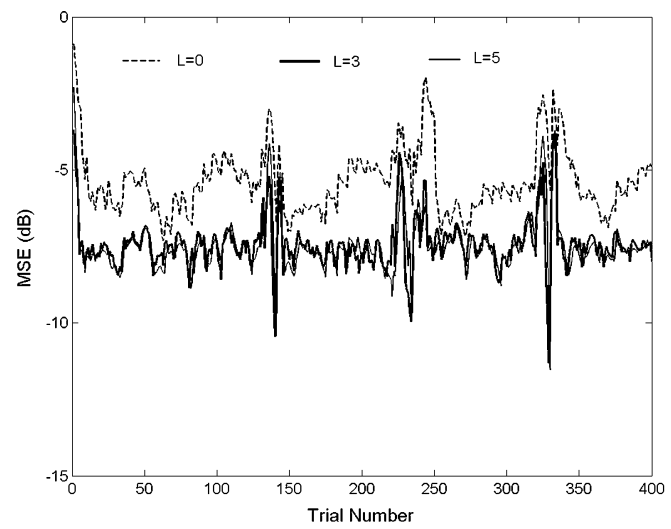

Fig. 5. MSE curves of DR-LMS with shown at three different settings of $L$. Note the conventional normalized LMS method is represented by $L=0$.

additional distortion because a large $L$ requires the use of more previous data. The old data will cause delays in tracking changes in the current signal. In fact, Fig. 5 shows that the tracking capacity of NDR-LMS with $L=5$ does not surpass that for $L=3$. Moreover, a large $L$ results in lengthy computation and may depress the signal-tracking capacity for nonstationary signals. In this simulation, a choice of $L=3$ produced an adequate estimate of the nonstationary EP signal.

Fig. 6 shows three-dimensional (3-D) views of EP signal tracking using previous RBFN [19] and the new DR-RBFN
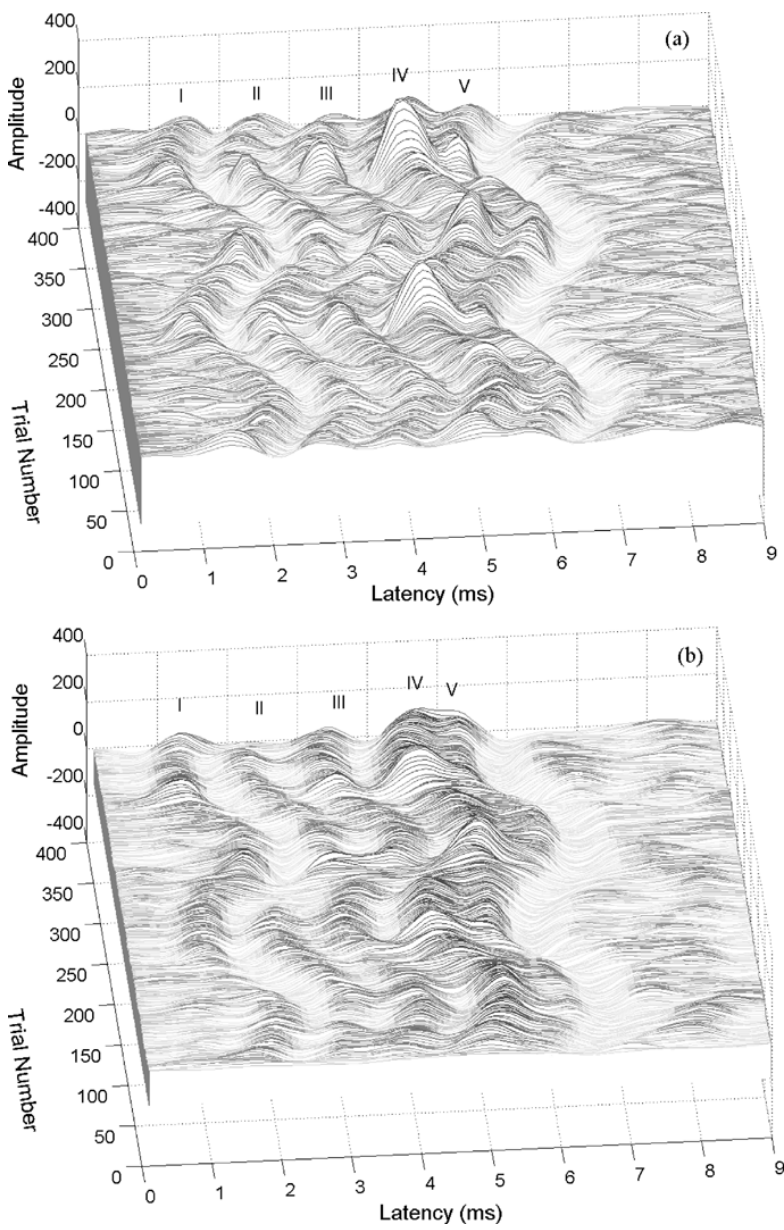

Fig. 6. Three-dimensional view of EP signal estimated respectively by (a) DR-RBFN, and (b) the previous RBFN algorithm.

algorithm. As expected the DR-RBFN produces better tracking of nonstationary signals. The data-reusing RBFN algorithm is especially suitable for estimating EP signals from data sets where a long recording time is not permitted, or for some applications such as the effects of drugs, where conditions are changing and long data sets may not be useful.

In EP measurement, both amplitude and latency estimations are equally important. The MSE values shown in Fig. 5 can only give a sketchy view of the performance of RBFN for EP estimation. The comparisons of the performance of algorithm on both amplitude and latency are given in Fig. 7. The simulated BAEP recordings are the same as described above. Fig. 7(a) shows latency tracking performance, and Fig. 7(b) presents amplitude tracking performance by both DR-RBFN and the previous RBFN. The bold lines in Fig. 7 are simulated latency shift and amplitude variation. The thin and dotted lines present estimated latency and amplitude by DR-RBFN and the previous $\mathrm{RBFN}$, respectively. It is clear that the tracking performance of new DR-RBFN markedly surpasses the previous RBFN, on tracking both latency shift and amplitude variations. It can also be seen that there are $2 \sim 4$ trials delay on tracking large amplitude variations even using new DR-RBFN.

From this simulation we have observed that the algorithm can track both latency and amplitude variation very well if variations are kind of "typical," i.e., change gradually across trials. It can be reasonably expected that if latency or amplitude is varied in other 

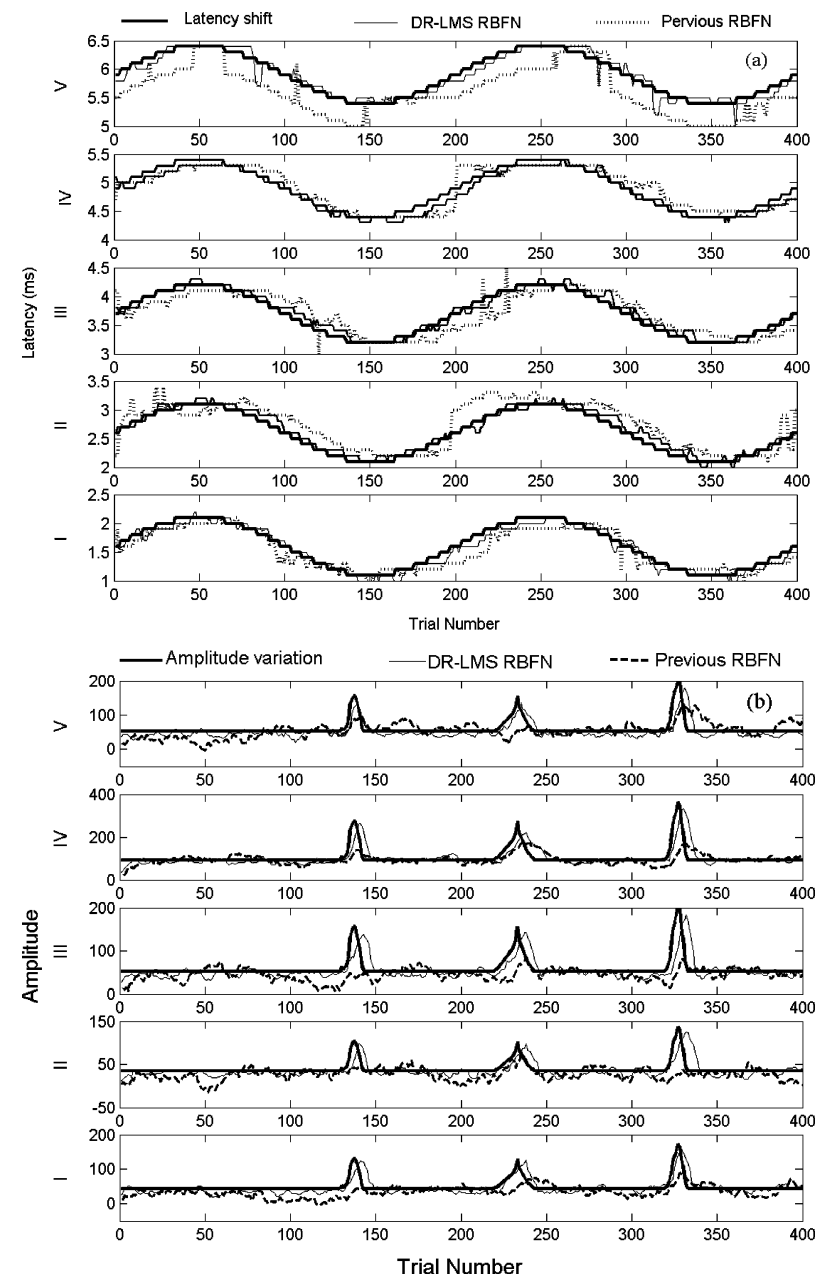

Fig. 7. Comparison of tracking performance on gradual variations of latency and amplitude of the two RBFN algorithms: (a) tracking curves on latency; (b) tracking curves on amplitude.

nonsinusoidal fashions (e.g., linear pattern), the algorithm tracks the signal variation well if the variation is gradual across trials.

\section{B. Performance Comparison at Different Noise Levels}

The simulated signal and EEG noise are the same as described above. Different SNRs for the simulated EP recordings were created to compare the filter performance for different noise levels. The SNR was varied from $-40 \mathrm{~dB}$ to $0 \mathrm{~dB}$ in increments of $5 \mathrm{~dB}$. In this example, the EP signal was deterministic across trials. The number of trials was 1000. The previous RBFN [19] and the present DR-RBFN algorithms were used to process the simulated recordings. The mean MSE across trials was calculated for each algorithm at different noise levels (Fig. 8). It is clear that the new DR-RBFN algorithm is better than the previous RBFN at all SNR levels. At the poorest SNR the new algorithm outperforms the old one by as much as $10 \mathrm{~dB}$.

\section{Tracking Performance Simulation 2 (Abrupt Changes)}

A total of 400 EP signals were simulated using the same EP signal and noise of the Simulation 1. Two abrupt changes were introduced in 400 trials of simulated EP recording. The first abrupt change appeared from trial 51 to 100 , when the signal amplitude was set to zero in order to simulate the disappearance of EP in a realistic situation. The second abrupt change occurred between trial 150 and 151, when the response

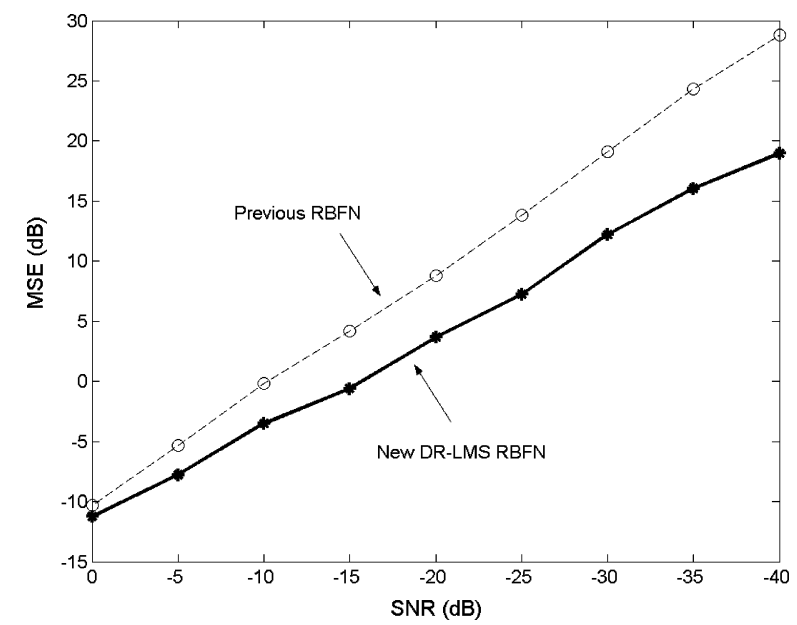

Fig. 8. Comparison of performance of the two RBFN algorithms at different noise levels. The SNR varied from $-40 \mathrm{~dB}$ to $0 \mathrm{~dB}$ in increments of $5 \mathrm{~dB}$. The number of trials was 1000 and the EP signal was deterministic across trials. The MSE values were calculated trial by trial and then were averaged to produce the plot.

latency was abruptly delayed by $2.5 \mathrm{~ms}$. In addition, two gradual changes were also included in this simulation: from trial 181 to 300 a linear latency shift was inserted, and from trial 220 to 231 the signal amplitude was amplified. The 400 trials of simulated BAEP signal with abrupt change are shown in Fig. 9(a). Fig. 9(b)-(c) represents EP signal estimates by the DR-DRBF and the previous RBFN respectively. It is clear that DR-RBFN outperforms the previous RBFN in both latency and amplitude estimations.

The details of tracing performance of two algorithms are presented in Fig. 10. Although there are some delays both algorithms could briefly follow abrupt and gradual changes well. Fig. 10 shows that DR-RBFN can track latency and amplitude variations faster and more accurately. Fig. 11 displays the outputs of two algorithms. The abrupt latency shift took place at trial 151 where the latency sharply shifted up $2.5 \mathrm{~ms}$. It is clear in the figure that at $\mathrm{i}=151$ and 152 both algorithms failed to respond fast enough to follow such an abrupt change because of the inertia of the weight which was converged to trial 150 . At the next two trials ( $\mathrm{i}=153,154)$, DR-RBFN adjusted its weights effectively to track the abrupt change on both latency and amplitude, while the previous RBFN could only follow the latency change.

The simulations showed the limitations of the algorithms. Even the response of DR-RBFN has $2 \sim 4$ trials in delay to the real change. If the abrupt changes occur quickly, it is hard for the algorithm to track change within one or two trials because of the inertia of weight. Whereas for gradual changes there is a long enough stable state after the start of change, the tracking of DR-RBFN becomes satisfactory.

\section{DATA ACQUisition AND PROCESSING}

In this section, the DR-RBFN algorithm described above is used to estimate human BAEP and intraoperative somatosensory evoked potential (SEP).

\section{A. BAEP}

A total of 2000 BAEP records were recorded from a subject, age 25. The stimulus used to evoke BAEP was a click, $0.1 \mathrm{~ms}$ 

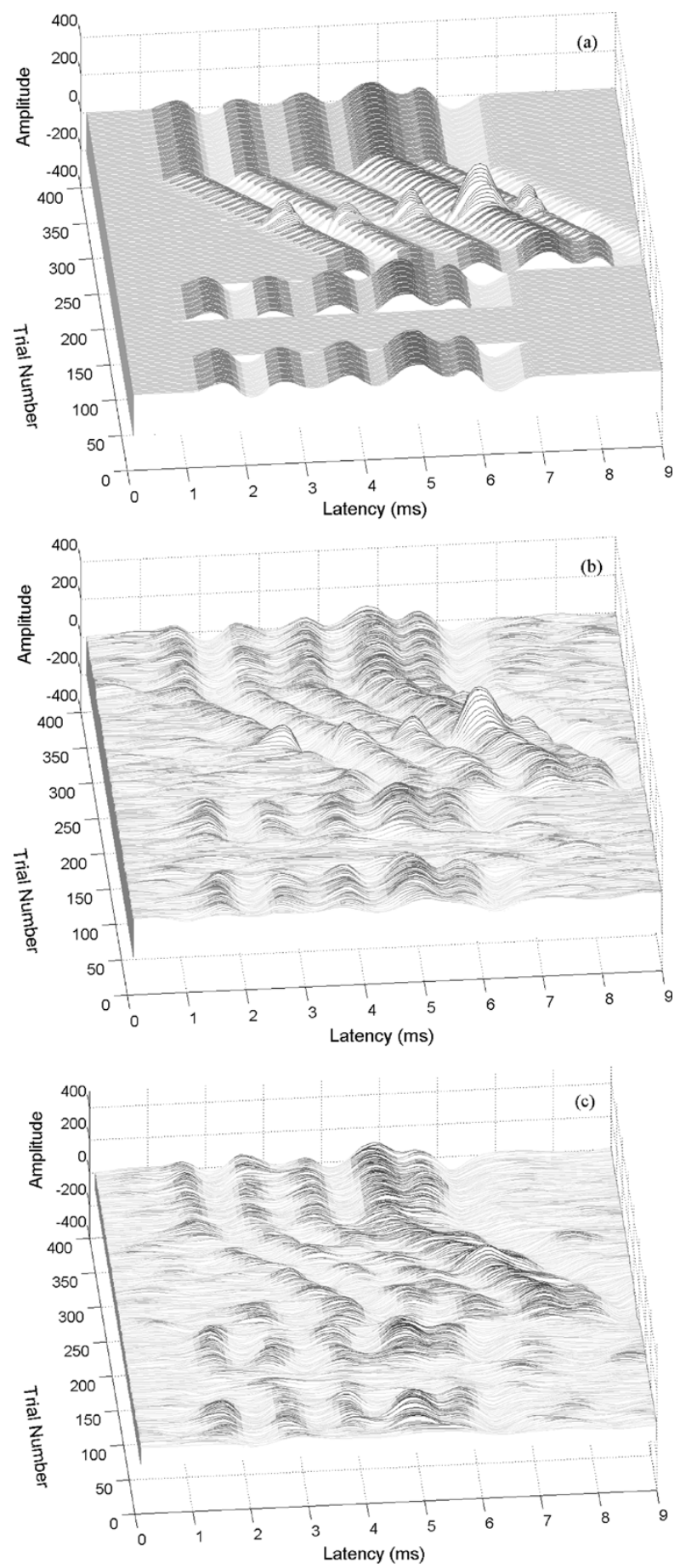

Fig. 9. Results on tracking latency and amplitude of artificially introduced abrupt changes in 3-D views: (a) simulated EP signals across 400 trials; (b) EP signals estimated by DR-RNBF; (c) EP signals estimated by the previous RBFN.

in duration, delivered at a rate of $10 / \mathrm{s}$, with $100-\mathrm{dB}$ peak sound pressure level. The response was recorded from the scalp surface with conventional electrode placement at the vertex and mastoid using a clinical EP machine (Nicolet) at a sampling rate of 10 $\mathrm{kHz}$. Conventional EP procedures such as electrical and acoustic shielding were used to reduce interference and noise. Only the first $9 \mathrm{~ms}$ of poststimulus data (corresponding to 90 data points at a sampling rate of $10 \mathrm{kHz}$ ) in each trial was processed for the early auditory response or BAEP. The DR-RBFN algorithm
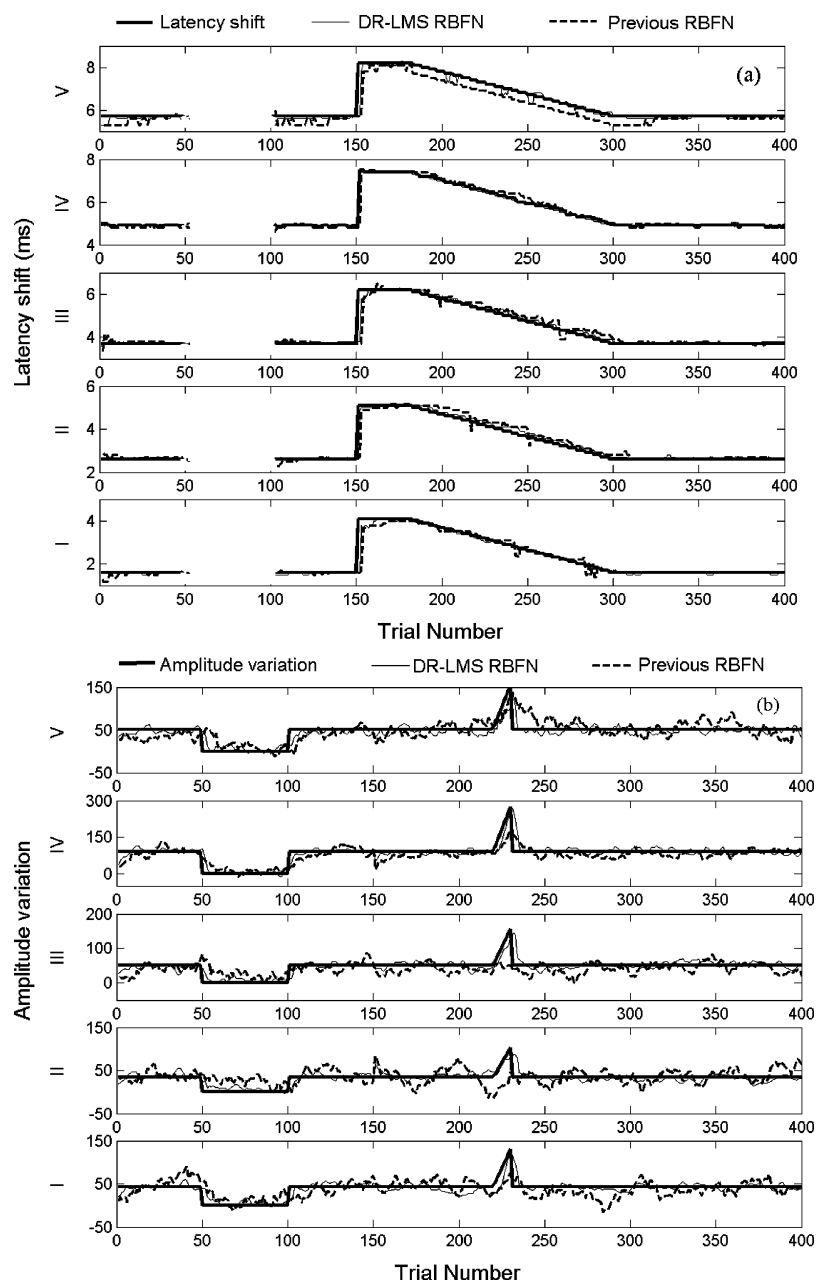

Fig. 10. Comparisons of tracking performance on abrupt changes using the two RBFN algorithms. Details of tracking curves on latency shift (a), amplitude variations (b).

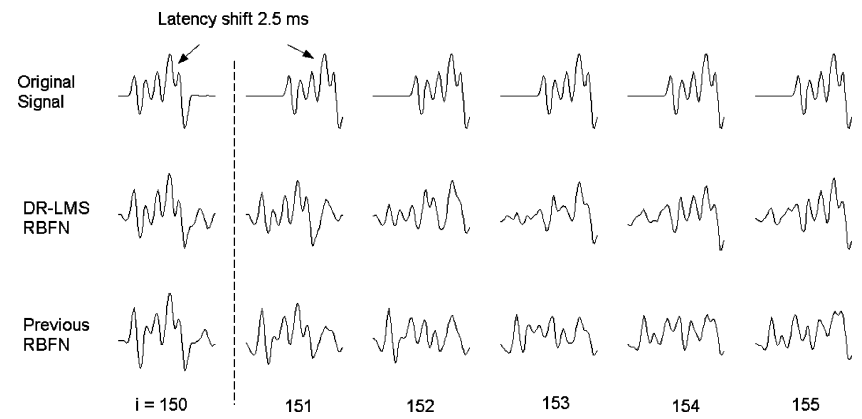

Fig. 11. Close looks on tracking of abrupt changes in EP using two RBFN algorithms. Note the DR-RBFN outperforms the previous RBFN on tracking both latency and amplitude variations.

(with $L=3$ ) was used to estimate the BAEP signal in real-time. Fig. 12 shows an isometric view of the BAEP across trials. It is clear that the BAEP signal varies in both amplitude and latency across trials.

\section{B. Intraoperative SEP}

SEP is the response of central nervous system to external stimulation. SEP has been routinely used for intraoperative monitoring to alarm surgeons of possible spinal cord compression and initiate interventions during neurosurgery. However, the inadequacy of 


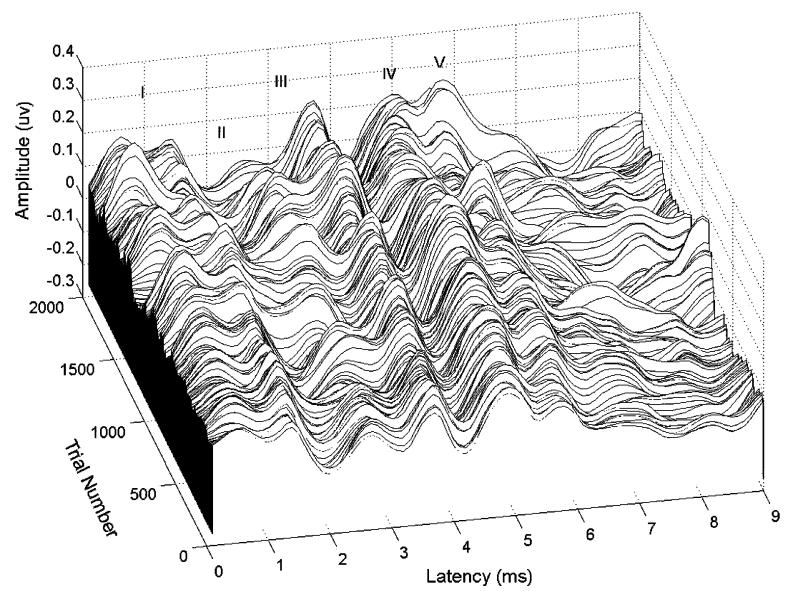

Fig. 12. Isometric view of the human BAEP single trial signals extracted by DR-RBFN

SEP for reliable monitoring of spinal cord functional has already been documented [29]-[32]. SEP relies on an averaging technique, which requires a period of $10-40 \mathrm{~s}$ or in worse cases 30 min for updating. Therefore, a reliable intraoperative spinal cord monitoring system with a fast SEP signal detection algorithm is most desirable for obvious reasons.

We applied DR-RBFN algorithm to measure SEP during orthopaedic surgery to treat scoliosis. Eight scoliosis patients (age 15 to 23) took part in this study. All patients received general anaesthesia (thiopental at $0.4 \mathrm{mg} / \mathrm{kg}$, or fentanyl at $1-2 \mu \mathrm{g} / \mathrm{kg}$ and maintained with isoflurane at $0.8 \%$ and nitrous oxide/oxygen: $60 \% / 40 \%$ ). To elicit SEP, a pair of stimulating electrodes was applied over the posterior tibial nerve behind the medial malleoli, with constant current stimulation $(\sim 10-30 \mathrm{~mA})$. Single pulse stimulation with a frequency between 5.1 and $5.7 \mathrm{~Hz}$ and duration of $300 \mu$ s was applied. SEP signals were collected over $\mathrm{Cz}^{\prime}(2 \mathrm{~cm}$ posterior to $\mathrm{Cz}$, 10-20 international system for EEG electrode placement) versus the $\mathrm{Fz}$ of the 10-20 system. The signal was amplified 100000 times with an isolated amplifier (SCXI-1120, National Instruments Co., Austin, TX), and bandpass filtered between 20 and $3000 \mathrm{~Hz}$. All the SEP signals were acquired with a data acquisition card (DAQcard-1200, National Instruments Co., Austin, TX) at a 12-bit resolution and a sampling rate of $5 \mathrm{kHz}$. A DR-RBFN algorithm with $N=50$ and $L=3$ was used to monitor the SEP trial by trial.

Fig. 13 shows the continuously recorded 200 trials of the SEP recordings from one of patients, while Fig. 14(a) demonstrates an example of the real-time SEP raw signal from this case. The raw data [Fig. 14(a)] looks like noise and it is difficult to identify the evoked response waveform [compared with the averaged SEP, Fig. 14(b)]. The DR-RBFN algorithm was used to extract the SEP from the same 200 raw recordings shown in Fig. 13. The result displayed in Fig. 15 shows the SEP signal extracted by DR-RBFN, in which the peak latency and amplitude can be readily measured. The result is quiet consistent with the EA waveform.

The validation of DR-RBFN SEP extraction was evaluated by the comparison between DR-RBFN results and conventional EA SEP. For the purposes of comparison, the SEP measurements were made at five different stages of surgery defined below [31].

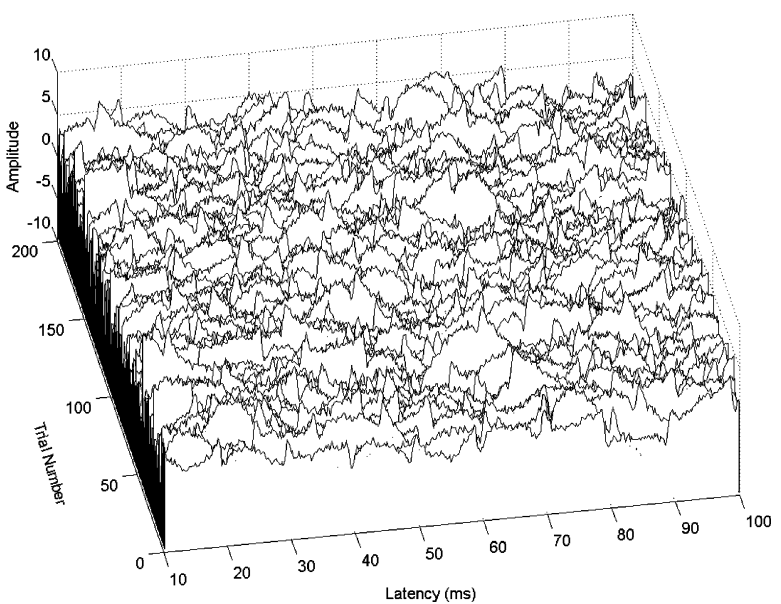

Fig. 13. Raw SEP recordings $(\mathbf{n}=200$ trials $)$ during scoliosis surgery in a human.

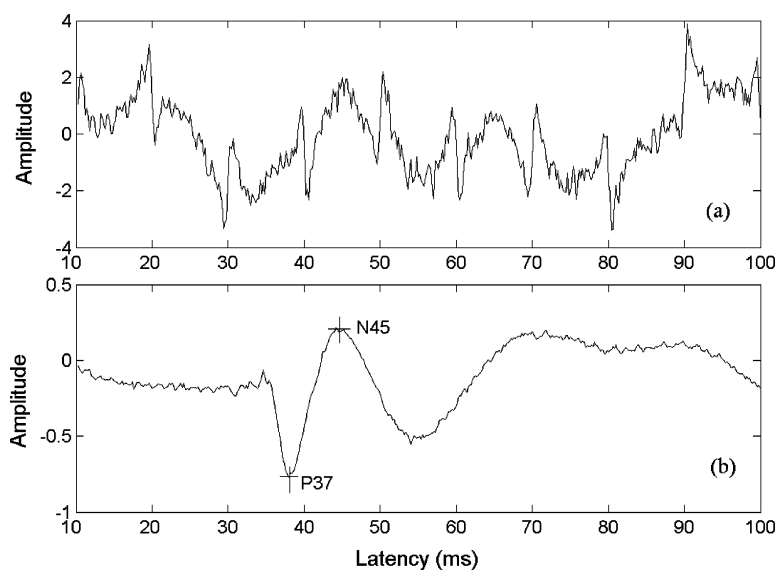

Fig. 14. (a) SEP signals showing an example of a single-trial raw SEP signal, and (b) the result of EA ( $\mathbf{n}=200$ trials $)$.

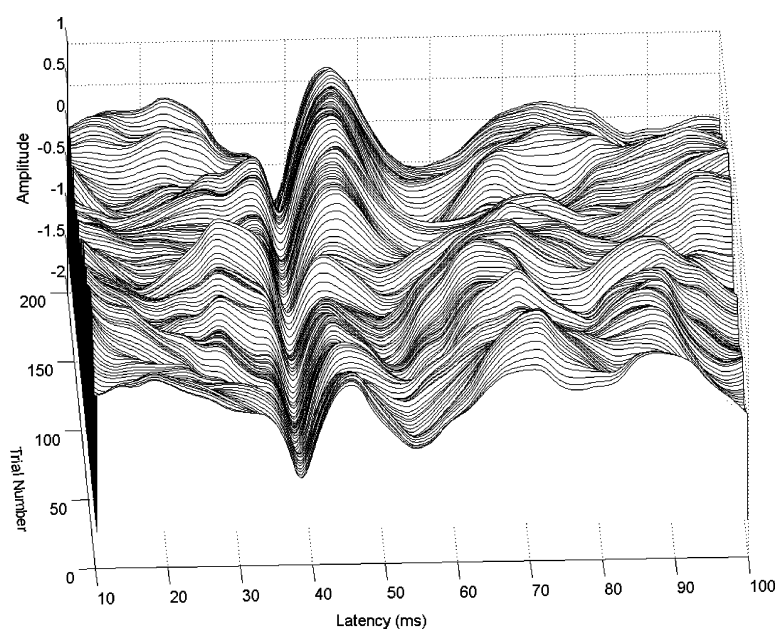

Fig. 15. SEP signals extracted by DR-RBFN algorithm.

Stage 1) (Preoperation) When the patient had been anaesthetized and positioned on the operating table.

Stage 2) (Spine exposure) When the muscle and soft tissues were stripped and the surgical field exposed.

Stage 3) (Instrumentation application) When the instrumentation, such as hooks, wires or screws were inserted. 
TABLE I

COMPARISON OF THE SEP MEASUREMENTS BETWEEN EA SEP AND DR-RBFN SEP

\begin{tabular}{|l|l|l|l|l|l|l|l|l|}
\hline & \multicolumn{2}{|l|}{ Amplitude $(\mu \mathrm{V})$} & \multicolumn{2}{l}{ Latency $(\mathrm{ms})$} & \multicolumn{2}{l|}{$\begin{array}{l}\text { Maximum \% changes of } \\
\text { amplitude vs stage 1 }\end{array}$} & $\begin{array}{l}\text { Maximum \% changes of } \\
\text { latency vs stage 1 }\end{array}$ \\
\hline & EA SEP & $\begin{array}{l}\text { DR-RBFN } \\
\text { SEP }\end{array}$ & EA SEP & $\begin{array}{l}\text { DR-RBFN } \\
\text { SEP }\end{array}$ & $\begin{array}{l}\text { EA SEP } \\
\text { DR-RBFN } \\
\text { SEP }\end{array}$ & EA SEP & $\begin{array}{l}\text { DR-RBFN } \\
\text { SEP }\end{array}$ \\
\hline Case 1 & $1.13 \pm 0.09$ & $1.19 \pm 0.09$ & $38.0 \pm 0.2$ & $38.2 \pm 0.2$ & $9.3 \%$ & $11.8 \%$ & $1.2 \%$ & $1.1 \%$ \\
\hline Case 2 & $1.93 \pm 0.09$ & $2.01 \pm 0.11$ & $38.3 \pm 0.1$ & $38.3 \pm 0.2$ & $12.4 \%$ & $12.1 \%$ & $1.4 \%$ & $1.9 \%$ \\
\hline Case 3 & $1.92 \pm 0.07$ & $2.13 \pm 0.12$ & $38.4 \pm 0.2$ & $38.3 \pm 0.1$ & $13.5 \%$ & $12.9 \%$ & $1.5 \%$ & $1.3 \%$ \\
\hline Case 4 & $0.91 \pm 0.05$ & $1.01 \pm 0.08$ & $38.1 \pm 0.4$ & $38.1 \pm 0.3$ & $11.6 \%$ & $17.5 \%$ & $2.7 \%$ & $2.1 \%$ \\
\hline Case 5 & $1.95 \pm 0.11$ & $2.12 \pm 0.09$ & $38.3 \pm 0.2$ & $38.2 \pm 0.2$ & $21.2 \%$ & $19.3 \%$ & $2.2 \%$ & $1.5 \%$ \\
\hline Case 6 & $2.08 \pm 0.12$ & $2.15 \pm 0.13$ & $38.7 \pm 0.3$ & $38.5 \pm 0.1$ & $15.7 \%$ & $15.8 \%$ & $2.1 \%$ & $1.4 \%$ \\
\hline Case 7 & $1.62 \pm 0.11$ & $1.72 \pm 0.10$ & $38.6 \pm 0.2$ & $38.5 \pm 0.2$ & $22.3 \%$ & $14.5 \%$ & $2.1 \%$ & $1.7 \%$ \\
\hline Case 8 & $1.41 \pm 0.10$ & $1.57 \pm 0.12$ & $38.4 \pm 0.3$ & $38.3 \pm 0.2$ & $13.7 \%$ & $12.6 \%$ & $1.8 \%$ & $1.3 \%$ \\
\hline
\end{tabular}
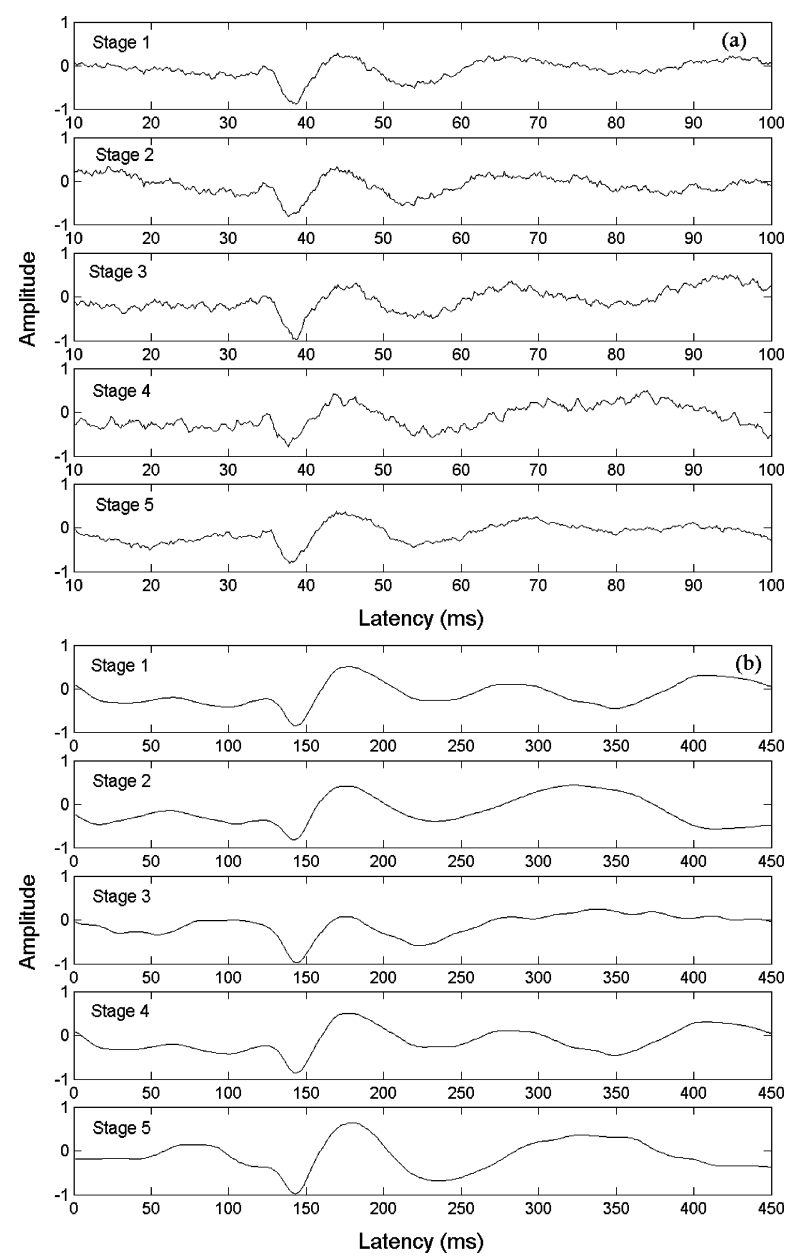

Fig. 16. (a) SEP in one patient obtained by EA during different surgical stages, in comparison with (b) SEP extracted by the DR-RBFN algorithm.

Stage 4) (Deformity correction) When the deformity was corrected by rod rotation, and compression or distraction.

Stage 5) (Wound closure) When the wound was closed.

At each stage we used 200 response trials to generate the conventional EA, and 30 trials to generate the DR-RBFN estimate of SEP. Thus, for each patient, there are two kinds of SEP estimates, one from EA (includes five EA SEP estimates, using a total 1000 trials), and another from DR-RBFN (including five DR-RBFN SEP estimates, using a total of 150 trials). Student's t-test was performed to assess statistical differences between the DR-RBFN and EA measurements. Fig. 16(a) shows an example of EA SEP in five surgical stages, while Fig. 16(b) shows the DR-RBFN SEP. In this case, the amplitude in different stages was measured $1.13 \pm 0.09 \mu \mathrm{V}$ in EA SEP, while was measured $1.19 \pm 0.09 \mu \mathrm{V}$ in DR-RBFN SEP. The latency in different stages was measured $38 \pm 0.2 \mathrm{~ms}$ in EA SEP, while was measured $38.2 \pm 0.2 \mathrm{~ms}$ in DR-RBFN SEP. In the other seven cases, similar comparison was performed.

Peak latencies and amplitudes were measured on each EA SEP and DR-RBFN SEP in different surgical stages. The results of five surgical stages in each case were statistically compared. Table I shows the amplitude/latency measurements by both EA SEP and DR-RBFN SEP. No significant difference was found between EA SEP and DR-RBFN SEP measurements ( $p>0.05$, Student's t-test). The SEP's recorded before any surgical procedures were treated as baselines which represented neurological normal condition. Percentage difference between the latency and amplitude of the baseline and later stages EA SEP as well as DR-RBFN SEP were calculated to show the variability. The criteria of abnormal SEP have not been established in consensus to define neural functional losses occurred during surgery [31], [32], [36]. The most commonly used criterion is either a reduction of control SEP peak amplitude of $>50 \%$ or a delay of peak latency of $>10 \%$ [31]-[35]. In this study, both EA SEP and DR-RBFN SEP showed similar results of variability, without significant difference in the comparison of percentage variability of both amplitude and latency $(\mathrm{p}>0.05$, Student's t-test). In both EA SEP and DR-RBFN SEP, the percentage variability of latency is $<3 \%$ and variability of amplitude $<$ $30 \%$, which are clinically considered subthreshold to neural function deficit.

DR-RBFN SEP may also provide trial to trial variability estimation, which need further study to investigate its clinical value in intraoperative spinal cord monitoring. Using DR-RBFN, SEP signal can be extracted from noisy background in less than 10 s using just a regular Pentium III PC, and can be faster with a better computer system or a specific DSP chip.

\section{CONClusion}

Adaptive algorithms are considered as suitable methods to estimate nonstationary signals. It is well recognized that the convergence rate is a very important criterion for these algorithms. In this paper, we have presented a data-reusing RBFN model for estimating EP signals in real-time. As a nonlinear system RBFN could provide a universal function approximation capability, which is more suitable for estimating nonstationary signals. The LMS algorithm is a simple and effective algorithm 
for real-time implementation of the RBFN. Moreover, Soni et al. [22] proved that the algorithms which jointly used NLMS and data reusing can achieve a fast convergence approximate to recursive least square techniques. The effectiveness of the DR-RBFN on EP extraction has been successfully demonstrated in this paper.

To accelerate the rate of convergence and, thus, enhance the tracking ability of nonstationary signals, a normalized data-reusing LMS algorithm was studied. The NDR-LMS algorithm reuses data pairs from previous iterations to generate the new gradient estimates that are in turn used to update the adaptive weight vector. This algorithm operates in real-time and has a fast convergence rate and can, thus, track EP signal variations across trials. In some situations, the EP signal must be estimated from only a few tens or 100 trials. The date-reusing RBFN is, thus, especially useful in the extraction of useful information from each trial. Comparison with either the traditional ensemble average or the previous RBFN [19] shows that the present method is more powerful in tracking EP variations. The relatively simple structure, powerful mapping capability and fast convergence features makes our new DR-RBFN algorithm a potentially valuable tool for estimating EP signals in both clinical and physiological research environments.

The RBF network used in this paper is a highly degenerated one, for it has only one fixed input and its kernels are predefined. The resulted structure is equivalent to a typical adaptive filter with a deterministic and periodic reference input. The qualitative analysis has been given in previous sections. In Section III, it has been shown that the algorithm can track relatively slow change of EP signal very well even at poor SNR. For abrupt change of the EP signal, it may take three to four trials to completely catch up with the variation. The convergence rate is the key for the successfully tracking the signal variation. Optimal design of RBFN, not just using a fixed simple Gaussian kernel, may improve the performance. Other promising algorithms such as affine projection algorithm could be our future work on EP signal extraction.

\section{APPENDIX}

The normalized LMS algorithm could be derived by the principle of minimal disturbance [26, pp. 321]. The problem is to minimize the squared Euclidean norm of the change

$$
\delta \mathbf{w}(k+1)=\mathbf{w}(k+1)-\mathbf{w}(k)
$$

subject to the constraint

$$
\mathbf{H w}(k+1)=\mathbf{d}(k) .
$$

Using the method of Lagrange multipliers, the problem can be solved by minimizing

$$
\begin{aligned}
F(k)=(\mathbf{w}(k+1)-\mathbf{w}(k))^{T}(\mathbf{w}(k+1)-\mathbf{w}(k)) \\
+\boldsymbol{\lambda}^{T}(\mathbf{d}(k)-\mathbf{H w}(k+1)) .
\end{aligned}
$$

Let

$$
\frac{\partial F(k)}{\partial \mathbf{w}(k+1)}=2(\mathbf{w}(k+1)-\mathbf{w}(k))-\mathbf{H}^{T} \boldsymbol{\lambda}=0
$$

we have

$$
\mathbf{w}(k+1)=\mathbf{w}(k)+\frac{1}{2} \mathbf{H}^{T} \boldsymbol{\lambda} .
$$

From (A2) and (A5) we have

$$
\mathbf{d}(k)=\mathbf{H w}(k+1)=\mathbf{H}\left(\mathbf{w}(k)+\frac{1}{2} \mathbf{H}^{T} \boldsymbol{\lambda}\right) .
$$

Then, from (5) and (A6) we get

$$
\boldsymbol{\lambda}=2\left(\mathbf{H H}^{T}\right)^{\dagger} \mathbf{e}(k) \text {. }
$$

Then, we get the solution of the constrained optimization problem as follows:

$$
\mathbf{w}(k+1)=\mathbf{w}(k)+\frac{1}{2} \mathbf{H}^{T} \boldsymbol{\lambda}=\mathbf{w}(k)+\mathbf{H}^{T}\left(\mathbf{H H}^{T}\right)^{\dagger} \mathbf{e}(k) .
$$

Consequently the normalized LMS algorithm

$$
\mathbf{w}(k+1)=\mathbf{w}(k)+\mu \mathbf{H}^{T}\left(\mathbf{H H}^{T}+\varepsilon \mathbf{I}\right)^{-1} \mathbf{e}(k)
$$

where $\mu$ is a positive real scaling factor to exercise control over the change in the weight vector, and $\varepsilon$ is a small positive constant helping to realize the pseudoinverse of the matrix.

\section{ACKNOWLEDGMENT}

The authors would like to thank Mr. B. Lam for his technical assistance.

\section{REFERENCES}

[1] T. Harmony, Neurometric Assessment of Brain Dysfunction in Neurological Patients. Hillsdale, NJ: Lawrence Erlbaum, 1984.

[2] C. D. Mcgillen, J. I. Aunon, and K. B. Yu, "Signals and noise in evoked brain potentials," IEEE Trans. Biomed. Eng., vol. BME-32, pp. 1012-1016, 1985.

[3] D. Regan, Human Brain Electrophysiology: Evoked Potentials and Evoked Magnetic Fields In Science and Medicine. New York: Elsevier, 1989.

[4] S. Nishida, M. Nakamura, and H. Shibasaki, "Method for single-trial recording of somatosensory evoked potentials," J. Biomed. Eng., vol. 15 , no. 3 , pp. $257-262,1993$.

[5] O. Ziegler, B. Guerci, M. Algan, P. Lonchamp, M. Weber, and P. Drouin, "Improved visual evoked potential latencies in poorly controlled diabetic patients after short-term strict metabolic control," Diabetes Care, vol. 17, no. 10, pp. 1141-1147, 1994.

[6] C. D. Woody, "Characterization of an adaptive filter for the analysis of variable latency neuroelectric signals," Med. Biol. Eng., vol. 5, pp. $539-553,1967$.

[7] J. I. Aunon and C. D. Mcgillem, "Measurements of signal components on single visually evoked brain potentials," IEEE Trans. Biomed. Eng., vol. BME-24, pp. 232-241, 1977.

[8] S. Cerutti, G. Chiarenza, D. Liberati, P. Mascellani, and G. Pavesi, "A parametric method of identification of single-trial event-related potentials in the brain," IEEE Trans. Biomed. Eng., vol. 35, no. 9, pp. 701-711, Sep. 1988.

[9] N. V. Thakor, "Adaptive filtering of evoked potentials," IEEE Trans. Biomed. Eng., vol. BME-34, pp. 6-12, 1987.

[10] P. G. Madhavan, "Minimal repetition evoked potentials by modified adaptive line enhancement," IEEE Trans. Biomed. Eng., vol. 39, no. 7, pp. 760-764, Jul. 1992.

[11] P. Laguna, R. Jane, O. Meste, P. W. Poon, P. Caminal, H. Rix, and N. V. Thakor, "Adaptive filter for event-related bioelectric signals using an impulse correlated reference input: Comparison with signal averaging techniques," IEEE Trans. Biomed. Eng., vol. 39, no. 10, pp. 1032-1044, Oct. 1992.

[12] X. H. Yu, Z. Y. He, and Y. S. Zhang, "Time-varying adaptive filters for evoked potential estimation," IEEE Trans. Biomed. Eng., vol. 41, no. 11, pp. 1062-1071, Nov. 1994.

[13] F. H. Y. Chan, F. K. Lam, P. W. F. Poon, and W. Qiu, "Detection of brainstem auditory evoked potential by adaptive filtering," Med. Biol. Eng. Comput., vol. 33, no. 1, pp. 69-75, 1995.

[14] F. H. Y. Chan, W. Qiu, F. K. Lam, P. W. F. Poon, and M. K. Lam, "Evoked potential estimation using modified time sequenced adaptive filter," Med. Biol. Eng. Comput., vol. 36, no. 4, pp. 407-414, 1998.

[15] W. Qiu, F. H. Y. Chan, F. K. Lam, and P. W. F. Poon, "Brainstem auditory evoked potential measurement using adaptive signal enhancement," Australasian Phys. Eng. Sci. Med., vol. 17, no. 3, pp. 131-135, 1994. 
[16] W. Qiu, K. S. M. Fung, F. H. Y. Chan, F. K. Lam, P. W. F. Poon, and R. P. Hamernik, " Adaptive filtering of evoked potentials with radial-basisfunction neural network prefilter," IEEE Trans. Biomed. Eng., vol. 49 no. 3, pp. 225-232, Mar. 2002

[17] A. Uncini, M. Marchesi, G. Orlandi, and F. Piazza, "Non-linear adaptive filter using neural network," in Proc. Int. Conf. Neural Network, vol. 1, 1990, p. 158.

[18] M. V. Shirvaikar and M. M. Trivedi, "A neural network filter to detect small targets in high clutter backgrounds," IEEE Trans. Neural Netw., vol. 6, no. 1, pp. 252-257, Jan. 1995.

[19] K. S. M. Fung, F. H. Y. Chan, F. K. Lam, and P. W. F. Poon, "A tracing evoked potential estimator," Med. Biol. Eng. Comput., vol. 37, no. 2, pp. 218-227, 1999.

[20] W. K. Jenkins, A. W. Hull, J. C. Strait, B. A. Schnaufer, and X. Li, Advanced Concepts in Adaptive Signal Processing. Boston, MA: Kluwer Academic, 1996.

[21] S. Roy and J. J. Shynk, "Analysis of the data-reusing algorithm," in Proc. 32nd Midwest Symp. Circuits and System, Champaign-Urbana, IL, 1989, pp. 1127-1130.

[22] R. A. Soni, K. A. Gallivan, and W. K. Jenkins, "Convergence properties of affine projection and normalized data reusing methods," in Proc. 32nd Asilomar Conf. Signal, Systems \& Computers, 1998.

[23] B. Widrow and S. D. Stearns, Adaptive Signal Processing. Englewood Cliffs, NJ: Prentice-Hall, 1985.

[24] J. Treichler and B. Agee, "A new approach to multipath correction of constant modulus adaptive algorithm," IEEE Trans. Acoust. Speech Signal Process., vol. ASSP-33, no. 4, pp. 402-431, 1983.

[25] T. Ku and C. Kuo, "Design and analysis of toeplitz preconditioners," IEEE Trans. Signal Process., vol. 40, no. 1, pp. 129-141, Jan. 1992.

[26] S. Haykin, Adaptive Filter Theory, 4th ed. Upper Saddle River, NJ: Prentice-Hall, 2002.

[27] S. T. Alexander, "Fast adaptive filters: A geometrical approach," IEEE Acoust., Speech, Signal Process. Mag., Oct. 1986.

[28] B. A. Schnaufer and W. K. Jenkins, "New data-reusing LMS algorithms for improved convergence," in Proc. 27th Asilomar Conf. Signal, Systems \& Computers, 1993.

[29] F. Sala, M. J. Krzan, and V. Deletis, "Intraoperative neurophysiological monitoring in paediatric neurosurgery: Why, when, how?," Childs Nerv. Syst., vol. 18, no. 6-7, pp. 264-287, 2002.

[30] K. Kothbauer, V. Deletis, and F. J. Epstein, "Intraoperative spinal cord monitoring for intramedullary surgery: An essential adjunct," Pediatr. Neurosurg., vol. 26, pp. 247-254, 1997.

[31] K. D. K. Luk, Y. Hu, Y. W. Wong, and J. C. Y. Leong, "Variability of somatosensory-evoked potentials in different stages of scoliosis surgery," Spine, vol. 24, no. 17, pp. 1799-1804, 1999

[32] K. D. K. Luk, Y. Hu, Y. W. Wong, and K. M. Cheung, "Evaluation of various evoked potential techniques for spinal cord monitoring during scoliosis surgery," Spine, vol. 26, no. 16, pp. 1772-1777, 2001.

[33] "American Electroencephalographic Society guidelines for intraoperative monitoring of sensory evoked potentials," J. Clin. Neurophysiol., vol. 4, no. 4, pp. 397-416, Oct. 1987.

[34] M. R. Nuwer, "Spinal cord monitoring with somatosensory techniques," J. Clin. Neurophysiol., vol. 15, no. 3, pp. 183-93, May 1998.

[35] — Evoked Potential Monitoring in the Operating Room. NY: Raven, 1986

[36] N. I. Spielholz, "Intraoperative monitoring using somato-sensory evoked potentials: A brief overview," Electromyogr. Clin. Neurophysiol., vol. 34, no. 1, pp. 29-34, Jan.-Feb. 1994

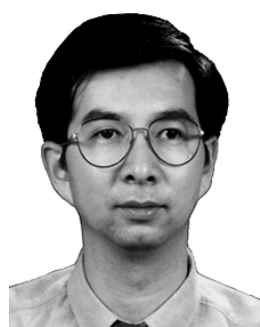

Wei Qiu (S'89-M'94) received the B.Sc. degree in biomedical engineering from Zhejiang University, Hangzhou, China, in 1984, and the M.Sc. and Ph.D. degrees in electronic engineering from South China University of Technology, Guangzhou, China, in 1991 and 1994 respectively.

He is currently Senior Research Scientist of Auditory Research Laboratories, State University of New York, Plattsburgh. He is also on the Faculty of the School of Electronic and Information Engineering, South China University of Technology. He was a Postdoctoral Research Fellow in the Department of Electrical and Electronic Engineering, Hong Kong University, Hong Kong, from 1996 to 1998. His research interests are in signal processing and their applications to biomedical engineering.

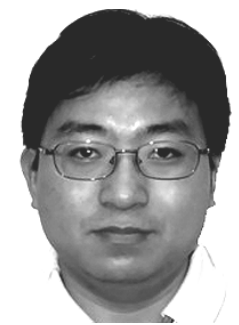

Chunqi Chang received the B.Sc. and M.Sc. degrees in electronic engineering from the University of Science and Technology of China, Hefei, China, in 1992 and 1995, respectively, and the Ph.D. degree in biomedical engineering from the University of Hong Kong, Hong Kong, in 2001.

He is currently a Postdoctoral Research Fellow in the Department of Electrical and Electronic Engineering at the University of Hong Kong. His main research interests are in statistical signal processing and their applications to biomedical engineering and computational molecular biology.

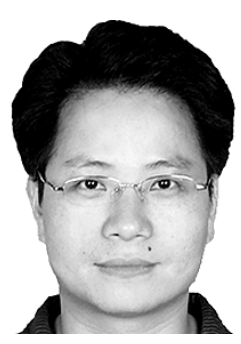

Wenqing Liu received the B.Sc. degree in Physics from Zhongshan University, Guangzhou, China, in 1985, and M.Sc. degree in electronics from Peking University, Peking, China, in 1988, and Ph.D. degree in electronic engineering in Hong Kong University, Hong Kong, in 2002. His research interests are in the area of biomedical signal processing.

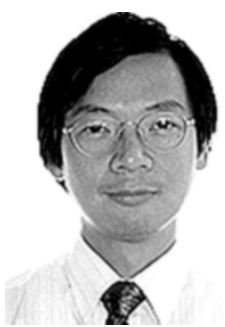

Paul W. F. Poon received the Ph.D. degree in neuroscience from Indiana University, Bloomington, IN, in 1979.

$\mathrm{He}$ served at the Department of Physiology, University of Hong Kong, Hong Kong, from 1979 to 1994 before moving to Taiwan. He is now Professor at the Department of Physiology, National Cheng Kung University, Tainan, Taiwan. His research interests, besides biomedical signal processing of humans, have included the neurophysiology of the central auditory system in processing complex sounds, pathophysiology of tinnitus, and experience-dependent neural plasticity in young animals.

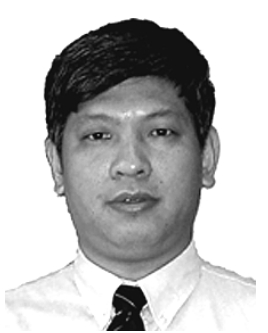

Yong $\mathrm{Hu}$ received the B.Sc. and M.Sc. degrees in the biomedical engineering from Tianjin University, Tianjin, China, in 1985 and 1988, respectively, the Ph.D. degree from The University of Hong Kong, Hong Kong, in 1999.

$\mathrm{He}$ is currently a Research Fellow and honorary Assistant Professor in the Department of Orthopaedics and Traumatology, Hong Kong University. $\mathrm{He}$ is also a distinguished Adjunct Professor in Institute of Biomedical Engineering, Chinese Academy of Medical Sciences, Shanghai, China, and Peking Union Medical College, Peking, China. His research interests include neural engineering, clinical electrophysiology, and biomedical signal measurement and processing.

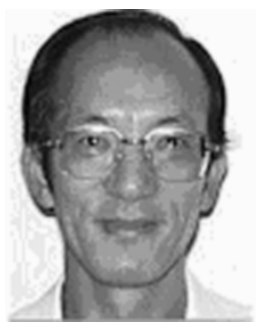

F. K. Lam obtained B.Sc. degree in engineering from the University of Hong Kong, Hong Kong, in 1965, and the M.Sc. and Ph.D. degrees from Loughborough University, Leicestershire, U.K., in 1969 and 1975, respectively. After working in Great Britain, he returned to Hong Kong University and now is Deputy Head of Department. His specialization is in signal processing and communication. 


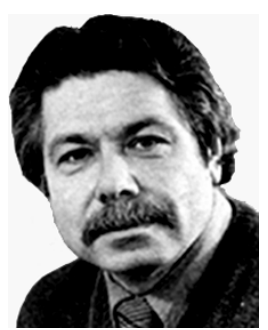

Roger P. Hamernik received the B.S., M.S., and Ph.D. degrees in mechanical engineering from Syracuse University, Syracuse, NY, in 1963, 1967, and 1970 , respectively.

$\mathrm{He}$ is currently Professor of Physics, and Speech and Hearing and Director of the Auditory Research Laboratories, State University of New York, Plattsburgh. From 1980 to 1985, he was a Professor with the Callier Certer for Communication Disorders, University of Texas at Dallas.

Dr. Hamernik is a Fellow of Acoustical Society of America. His research interests are in the area of auditory sciences.

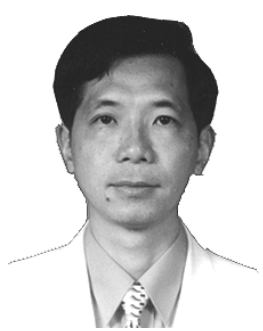

Gang Wei received the B.Sc. and M.Sc. degrees from Tsinghua University, Beijing, China, and the Ph.D. degree from South China University of Technology, Guangzhou, China, in 1984, 1987, and 1990, respectively. He was a Visiting Scholar at University of Southern California, Los Angeles, from June 1997 to June 1998.

$\mathrm{He}$ is currently a Professor in the School of Electronic and Information Engineering, South China University of Technology. His research interests are in signal processing and communications.

$\mathrm{He}$ is a Committee Member of the National Natural Science Foundation of China.

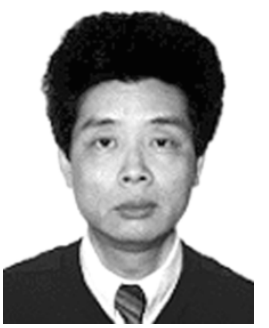

Francis H. Y. Chan received the Ph.D. degree from Bristol University, Bristol, U.K., in 1976.

He joined the Electrical and Electronic Engineering Department, The University of Hong Kong, Hong Kong, in 1976. Currently, he is Professor and Director of the University of Hong Kong Biomedical Engineering Research Centre. His research interest is in biomedical signal processing. 